\title{
On the Finite Volume Multigrid Method: Comparison of Intergrid Transfer Operators
}

\author{
K. S. Kang
}

\begin{abstract}
In this paper, we consider finite volume multigrid methods on triangular meshes with control volume based intergrid transfer operators. We review the error analysis of the finite volume methods and the convergence analysis on the multigrid method. For several different triangulations, we investigate the error reduction factors of the multigrid method as a solver, and also as a preconditioner in the Preconditioned CGM and GMRES solvers. We also study the scaling properties of the finite volume multigrid method on a High Performance Computer. We identify that the intergrid transfer operator based on the trial function space has the best properties.
\end{abstract}

2010 Mathematical subject classification: 65N08; 65N55; 65Y05.

Keywords: Finite Volume Method; Multigrid Method; Preconditioner .

\section{Introduction}

A finite volume method is a preferred method for the computational fluid dynamics (CFD) researcher because it has energy conservative properties which are required for CFD problems and are hard to ensure by a finite element method $[7,8,19]$. Numerical analysis of the finite volume method is more difficult than that of the finite element method, because, in general, the finite volume method uses two different function spaces: a trial space and a test space. To overcome the difficulty of such an analysis, many authors use the comparison analysis of the finite element method with a one-to-one corresponding relations between function spaces $[9,10,11,13,18]$.

Multigrid methods are well known as an efficient solution technique for many problems including elliptic and hyperbolic partial differential equations, non-linear problems, and even systems of algebraic equations which are not derived from any spatial discretization of a partial differential equation $([1,3,4,5,6,14,15,17,20])$. The number of operations of the multigrid method depends on the number of degrees of freedom (DoF) times the number of levels (log of the number of DoF). In many areas the multigrid method is regarded as an essential algorithm and it is applied to many different problems.

The multigrid algorithm of the linear finite element method on triangle meshes is a well analyzed problem $[2,4]$. Because the discretized linear system of the finite volume method for the Poisson problem is the same as for the finite element method (with a different right hand side) and the convergence of the finite element multigrid method is well analyzed, few authors consider the convergence analysis of the finite volume multigrid method [12]. It can be easily seen that the analysis result of the finite element multigrid method applies to the

K. S. Kang

High Level Support Team (HLST), Department of Computational Plasma Physics, Max-Planck-Institut für Plasmaphysik, Boltzmannstraße 2 D-85748 Garching bei München, Germany

E-mail: kskang@ipp.mpg.de. 
finite volume multigrid method with the natural injection on the trial function spaces as an intergrid transfer operator. Instead of the natural injection, we can use different intergrid transfer operators as in [21] for vertex-centered and cell-centered methods on rectangular grid and as in [5] for the linear finite element discretization. Another approach to define intergrid transfer operators for the finite volume multigrid method is based on the test function spaces which are defined on dual partitions, i.e., control volumes. This approach on the $P_{1}$ nonconforming finite element space has good properties to apply for non-linear problems, but has a poor convergence reduction factor [17]. The test function spaces of the finite volume methods are not nested themselves, so the multigrid method with the control volume based intergrid transfer operators was analyzed in different approaches $([12,16,17])$.

In this paper, we consider two different intergrid transfer operators on triangular meshes which are based on the test function spaces of the non-overlapping and overlapping finite volume methods. We compared these two intergrid transfer operators with the natural injection operator which is based on the trial function space. The multigrid method with the intergrid transfer operators based on the trial function space has the best convergence rate, except on a uniform mesh on which the multigrid method with the non-overlapping control volume based intergrid transfer operator has a better convergence rate.

Nowadays massively parallel computers are used to solve and/or simulate huge problems. By the nature of the multigrid method and the nature of parallelization, the W-cycle and variable $\mathrm{V}$-cycle multigrid methods are not preferred to be used on massively parallel computers. On the other hand the V-cycle multigrid method is widely used and achieves very good scaling properties. The scaling properties of the multigrid method highly depends on the system architecture. In this paper, we investigate them for the finite volume multigrid method on the HPC-FF machine at the Jülich Supercomputer Center (JSC).

This paper is organized as follows. The finite volume method and its error analysis results is summarized in section 2. In section 3, Two intergrid transfer operators are considered and the results of the convergence analysis for the finite volume multigrid method are summarized. In section 4, we report the results of numerical experiments of the multigrid method as a solver and as a preconditioner of the Krylov subspace methods and show the scaling properties of the multigrid method on the HPC-FF machine.

\section{Model problem and its discretizations}

In this section, we review the finite volume element (FVE) method on a general triangular mesh. The FVE method can be explained by the trial and the test function spaces. Usually, the trial function space is defined on a primal triangulation and the test function space is defined on a dual triangulation (control volume). Depending on the definition of the control volumes, the FVE methods are classified as non-overlapping and overlapping methods.

Let $H^{s}(\Omega)$ be the usual Sobolev spaces with $L^{2}$-norm where $s$ is a non-negative integer. Let $(\cdot, \cdot)$ denote the $L^{2}(\Omega)$ and $\|\cdot\|_{s}$ and $|\cdot|_{s}$ denote the $H^{s}$ norm and semi-norm respectively. As usual, $W^{k, \infty}$ is the Sobolev space with $L^{\infty}$-norm.

We consider a second-order elliptic problem with the Dirichlet boundary condition

$$
\begin{aligned}
-\nabla \cdot \mathbb{A} \nabla u & =f, & & \text { in } \Omega, \\
u & =0, & & \text { on } \partial \Omega,
\end{aligned}
$$

where $\Omega \in \mathbb{R}^{2}$ is a bounded polygonal domain with boundary $\partial \Omega, f \in L^{2}(\Omega)$, and the symmetric coefficient matrix $\mathbb{A} \in\left(W^{2, \infty}(\Omega)\right)^{2 \times 2}$ is uniformly elliptic, i.e., there exist positive 


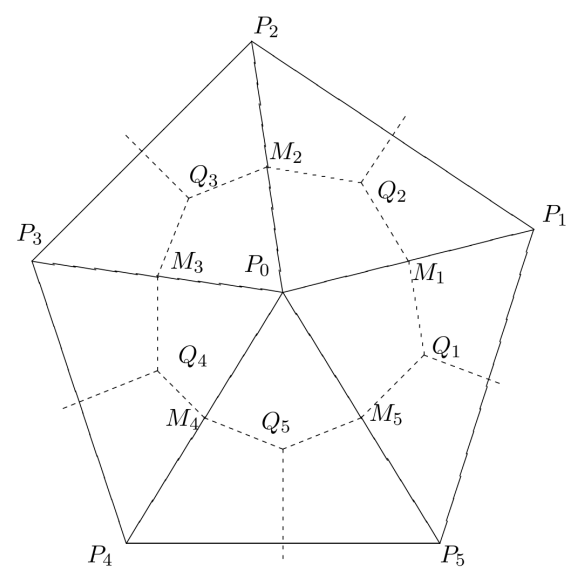

Figure 1. The primal and dual triangulations.

constants $\alpha_{0}, \alpha_{1}>0$ such that

$$
\alpha_{0} \chi^{t} \chi \leqslant \chi^{t} \mathbb{A}(x, y) \chi \leqslant \alpha_{1} \chi^{t} \chi, \quad(x, y) \in \Omega, \chi \in \mathbb{R}^{2} .
$$

It is well known that (1) has a unique solution.

By taking an integral of (1) over any control volume $V \in \bar{\Omega}$ with a Lipschitz boundary and using the Gauss Divergence Theorem on the left-hand side, (1) is transformed to the following primitive form (or surface integral form):

Find $u \in H^{1}(\Omega)$ such that, for any volume $V \in \bar{\Omega}$ with the Lipschitz boundary,

$$
-\int_{\partial V}(\mathbb{A} \nabla u) \cdot \mathbf{n} \mathrm{d} \sigma=\int_{V} f \mathrm{~d} x
$$

where $\mathbf{n}$ is an outward normal unit vector on $\partial V$.

In general, discretization based on the primitive form can preserve some conservation law. This is quite important in CFD, and is one of the reasons for its popularity.

First, we consider the primal discretization on which the trial function space is defined. Let $h_{0}$ and $\mathcal{T}_{h_{0}} \equiv \mathcal{T}_{0}$ be given, where $\mathcal{T}_{0}$ is a partition of $\Omega$ into triangles and $h_{0}$ is the maximum diameter of the elements of $\mathcal{T}_{0}$. For each integer $1 \leqslant k \leqslant J$, let $h_{k}=2^{-k} h_{0}$ and the sequence of triangulations $\mathcal{T}_{h_{k}} \equiv \mathcal{T}_{k}$ be constructed by the nested-mesh subdivision method, i.e., let $\mathcal{T}_{k}$ be constructed by connecting the midpoints of the edges of the triangles in $\mathcal{T}_{k-1}$, and let $\mathcal{T}_{h_{J}} \equiv \mathcal{T}_{J}$ be the finest grid. We define the trial function spaces as the piecewise linear finite element spaces

$$
V_{k}=\left\{v \in C^{0}(\Omega):\left.v\right|_{K} \text { is linear for all } K \in \mathcal{T}_{k}\right\},
$$

for $k=0, \ldots, J$.

Next, we construct the non-overlapping dual partition $\mathcal{T}_{k}^{*, n}$ by choosing any interior point $Q_{K}$ of $K \in \mathcal{T}_{k}$ and connect it with the mid-points of edges of $K$. In this paper, we are particularly interested in the case when $Q_{K}$ is the circumcenter (which are the respective midpoint of the either circumscribed circle of $K$ ) or centroid (intersection of its medians) of the triangle $K$. The dual partition associated with the circumcenter of the triangle forms the so-called Voronoi mesh and the dual partition with the centroid is in common use in CFD and 
forms the so-called Donald mesh. In order to keep the circumcenter from lying outside of the triangle, we assume that no interior angle of any triangle in $\mathcal{T}_{k}$ is larger than $90^{\circ}$. Carrying out the construction control volumes $K_{P}^{*, n}$ which is the surrounded area by connecting the mid-point of edge and $Q_{K}\left(M_{1} Q_{2} M_{2} Q_{3} M_{3} Q_{4} M_{4} Q_{5} M_{5} Q_{1} M_{1}\right.$ for $P_{0}$ in figure 1) for every node in the primal partition, we obtain the dual partition for the domain as $\mathcal{T}_{k}^{*, n}=\cup K_{P}^{*, n}$. Another approach is the overlapping dual partition which integrates all triangles which have $P_{0}$ as a vertex, i.e., the area surrounded by $P_{1} P_{2} P_{3} P_{4} P_{5} P_{1}$ in figure 1 . We denote the control volume of node $P$ by $K_{P}^{*, o}$ and the dual partition by $\mathcal{T}_{k}^{*, o}=\cup K_{P}^{*, o}$.

To define the non-overlapping FVE problem, we need a finite set of the control volumes $\mathcal{T}_{k}^{*, n}$ which has the same cardinality of $V_{k}$. Then the discrete FVE problem of (2) can be written as follows: Find $u_{h}^{*, n} \in V_{k}$ such that, for all $K_{P}^{*, n} \in \mathcal{T}_{k}^{*, n}$,

$$
-\int_{\partial K_{P}^{*, n} \backslash \partial K_{P}^{*, n} \cap \partial \Omega}\left(\mathbb{A} \nabla u_{k}^{*, n}\right) \cdot \mathbf{n} \mathrm{d} \sigma=\int_{K_{P}^{*, n}} f \mathrm{~d} x .
$$

We define associated test function spaces $Y_{k}^{*, n}$ as the space of piecewise constant functions:

$$
Y_{k}^{*, n}=\left\{z \in L^{2}(\Omega):\left.z\right|_{K_{P}^{*, n}} \text { is a constant function for all } K_{P}^{*, n} \in \mathcal{T}_{k}^{*, n}\right\} .
$$

Obviously, we have

$$
Y_{0}^{*, n} \not \subset Y_{1}^{*, n} \not \subset \cdots \not \subset Y_{J}^{*, n} .
$$

Define bilinear forms on the spaces $V_{k} \times Y_{k}^{*, n}$, for each $k$, as follows,

$$
\begin{aligned}
b_{k}^{*, n}\left(u_{k}, v_{k}\right) & =-\sum_{K_{P}^{*, n} \in \mathcal{T}_{k}^{*, n}} \int_{\partial K_{P}^{*, n} \backslash \partial K_{P}^{*, n} \cap \partial \Omega}\left(\mathbb{A} \nabla u_{k}\right) \cdot \mathbf{n} v_{k} \mathrm{~d} \sigma \\
& =-\sum_{K_{P}^{*, n} \in \mathcal{T}_{k}^{*, n}} v_{k}(P) \int_{\partial K_{P}^{*, n} \backslash \partial K_{P}^{*, n} \cap \partial \Omega}\left(\mathbb{A} \nabla u_{k}\right) \cdot \mathbf{n} \mathrm{d} \sigma .
\end{aligned}
$$

Then the non-overlapping finite volume discretization equation of (1) can be written as: Find $u_{J}^{*, n} \in V_{J}$ such that

$$
b_{J}^{*, n}\left(u_{J}^{*, n}, v_{J}\right)=F^{*, n}\left(v_{J}\right)=\left(f, v_{J}\right), \quad \text { for all } v_{J} \in Y_{J}^{*, n} .
$$

To define the overlapping FVE problem, we have the following relation when $\mathbb{A}$ is the identity matrix

$$
-\int_{\partial K_{P}^{*, o}}\left(\mathbb{A} \nabla u_{k}^{*, o}\right) \cdot \mathbf{n} \mathrm{d} \sigma=\frac{2}{3} \int_{K_{P}^{*, o}} f \mathrm{~d} x .
$$

But, we need to modify the bilinear form [9] for general $\mathbb{A}$. The discrete FVE can be written as follows: Find $u_{h}^{*, o} \in V_{k}$ such that, for all $K_{P}^{*, o} \in \mathcal{T}_{k}^{*, o}$,

$$
-\int_{\partial K_{P}^{*, o}}\left(\mathbb{A} \nabla u_{k}^{*, o}\right) \cdot \mathbf{n} \mathrm{d} \sigma+\frac{1}{2} \sum_{e \in E_{k}^{*, o}\left(K_{P}^{*, o}\right)} \int_{e}\left[\mathbb{A} \nabla u_{k}^{*, o}\right] \cdot \mathbf{n}_{e} \mathrm{~d} \sigma=\int_{K_{P}^{*, o}} f \mathrm{~d} x
$$

where

$$
[\mathbb{A} \nabla w] \cdot \mathbf{n}_{e}=\left(\mathbb{A} \nabla w_{K^{+e}}\right) \cdot \mathbf{n}_{e}-\left(\mathbb{A} \nabla w_{K^{-e}}\right) \cdot \mathbf{n}_{e},
$$

with $K^{+e}, K^{-e}$ denoting two triangles having interior edge $e$ as a common edge and $\mathbf{n}_{e}$ the normal vector of $e \in E_{k}^{*, o}\left(K^{+e}\right)$, and $E_{k}^{*, o}\left(K_{P}^{*, o}\right)=\{e$ is an edge $: P \in e\}$ in figure 2. 

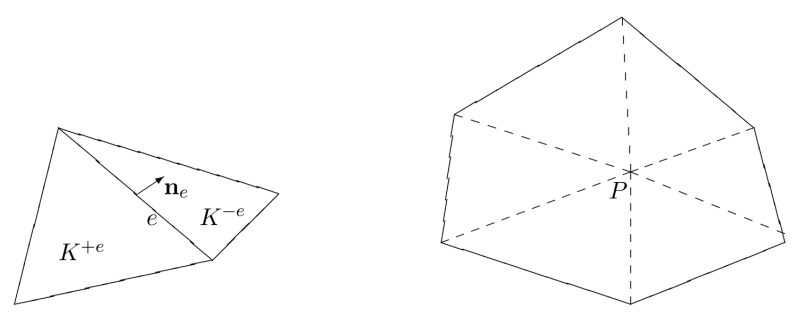

Figure 2. The triangles $K^{+e}$ and $K^{-e}$ with common side $e$ and $E_{k}^{*, o}\left(K_{P}^{*, o}\right)$ with dotted line.

Let test function space $Y_{k}^{*, o}$ be the space of piecewise constant functions on $K_{P}^{*, o} \in \mathcal{T}_{k}^{*, o}$, i.e., for all $v \in Y_{k}^{*, o}$, we have

$$
v=\sum_{K_{P}^{*, o} \in \mathcal{T}_{k}^{*, o}} v(P) \chi_{K_{P}^{*, o}}
$$

where $\chi_{K_{P}^{* o}}$ is a characteristic function associated with the control volume $K_{P}^{*, o}$. Then define bilinear forms on the spaces $V_{k} \times Y_{k}^{*, o}$, for each $k$, as follows,

$$
b_{k}^{*, o}\left(u_{k}, v_{k}\right)=\sum_{K_{P}^{*, o} \in \mathcal{T}_{k}^{*, o}} v_{k}(P)\left\{-\int_{\partial K_{P}^{* o o}}\left(\mathbb{A} \nabla u_{k}^{*, o}\right) \cdot \mathbf{n} \mathrm{d} \sigma+\frac{1}{2} \sum_{e \in E_{k}^{*, o}\left(K_{P}^{*, o}\right)} \int_{e}\left[\mathbb{A} \nabla u_{k}^{*, o}\right] \cdot \mathbf{n}_{e} \mathrm{~d} \sigma\right\}
$$

Then the overlapping finite volume discretization equation of (1) can be written as: Find $u_{J}^{*, o} \in V_{J}$ such that

$$
b_{J}^{* o o}\left(u_{J}^{*, o}, v_{J}\right)=F^{*, o}\left(v_{J}\right)=\frac{1}{3}\left(f, v_{J}\right), \quad \forall v_{J} \in Y_{J}^{*, o} .
$$

From (3) and (4), we can write same notation for the non-overlapping and overlapping $\mathrm{FVE}$ and denote by $*$ both $*, n$ and $*, o$ if not specified.

We introduce the one-to-one transfer operators $\gamma_{k}^{n}: V_{k} \rightarrow Y_{k}^{*, n}$ and $\gamma_{k}^{o}: V_{k} \rightarrow Y_{k}^{*, o}$ by

$$
\gamma_{k}^{n} v_{k}(x)=\sum_{j=1}^{N_{k}} v_{k}\left(P_{j}\right) \chi_{j}^{*, n}(x), \quad \gamma_{k}^{o} v_{k}(x)=\sum_{j=1}^{N_{k}} \frac{1}{3} v_{k}\left(P_{j}\right) \chi_{j}^{*, o}(x), \quad \forall x \in \Omega,
$$

where $P_{j}$ is a nodal point of the domain and $\chi_{j}^{*}$ is a characteristic function associated with the dual element $K_{P_{j}}^{*} \in \mathcal{T}_{k}^{*}, j=1, \ldots, N_{k}$. Then we can define a bilinear form

$$
a_{k}^{*}(v, w)=b_{k}^{*}\left(v, \gamma_{k} w\right) \quad \text { for all } v, w \in V_{k}
$$

and rewrite (3) and (4) as

$$
a_{J}^{*}\left(u_{J}^{*}, v_{J}\right)=F^{*}\left(\gamma_{J} v_{J}\right), \quad \forall v_{J} \in V_{J} .
$$

Remark 2.1. Let $a_{k}(v, w)=\sum_{K \in \mathcal{T}_{k}}(\mathbb{A} \nabla v, \nabla w)_{K}$ be a bilinear form over the spaces $H^{1}(\Omega)$ and $V_{k}$ by the finite element method. If $\mathbb{A}$ is piecewise constant, the we have, for all $v, w \in V_{k}$,

$$
a_{k}(v, w)=a_{k}^{*}\left(v, \gamma_{k} w\right),
$$

i.e., the system from the finite element method and the finite volume element methods, both the non-overlapping and the overlapping, are only different in the source term (right-hand side of equation). 


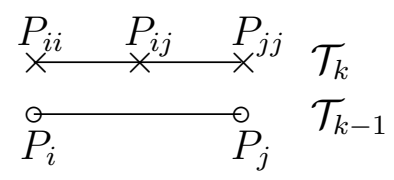

Figure 3. Notation for the intergrid transfer operator.

Using the analysis presented in [9] and [13], we get the following error estimations for the non-overlapping and overlapping FVE method.

Theorem 2.1. If $u \in H^{2}(\Omega)$ and $u_{J}^{*}$ are the solutions of (2) and (5). Then a constant $C$ exists, independent of $h_{J}$, such that

$$
\left\|u-u_{J}^{*}\right\|_{a_{J}} \leqslant C h_{J}\|u\|_{2}
$$

where $\|\cdot\|_{a_{J}}$ is a energy norm with the bilinear form $a_{J}(\cdot, \cdot)$.

Furthermore, assume that $f \in H^{1}(\Omega)$ and $\mathbb{A} \in W^{2, \infty}$. Then a constant $C$ exists, independent of $h_{J}$, such that

$$
\left\|u-u_{J}^{*}\right\|_{0} \leqslant C h_{J}^{2}\left(\|u\|_{2}+\|f\|_{1}\right) .
$$

\section{Intergrid transfer operators and convergence of the multigrid methods}

In this section, we consider the intergrid transfer operators for the finite volume multigrid method. Then we review the convergence analysis results of the multigrid method with these intergrid transfer operators.

First, we consider the case of the trial function spaces $V_{k}$. It is well known that the piecewise linear finite element spaces $V_{k}$ 's are nested and there are natural injection operators $I_{k}: V_{k-1} \rightarrow V_{k}$.

To define the intergrid transfer operator $I_{k}$, we denote by $P_{i}$ the vertex of $\mathcal{T}_{k-1}$ and by $P_{i j}$ the vertex of $\mathcal{T}_{k}$. The vertex $P_{i}$ of $\mathcal{T}_{k-1}$ is a vertex of $\mathcal{T}_{k}$, so we denote it by $P_{i i}$ in $\mathcal{T}_{k}$ as shown in figure 3. The mid-point of the edge $P_{i} P_{j}$ in $\mathcal{T}_{k-1}$ is a vertex of $\mathcal{T}_{k}$ and is denoted by $P_{i j}$ as shown in figure 3 .

From the definition of $V_{k}$ and the fact that $V_{k-1} \subset V_{k}$, we have

$$
I_{k} v\left(P_{i j}\right)=\left\{\begin{array}{l}
v\left(P_{i}\right), \quad \text { if } i=j \\
\frac{1}{2}\left(v\left(P_{i}\right)+v\left(P_{j}\right)\right), \quad \text { if } i \neq j
\end{array} .\right.
$$

Then we have

$$
a_{k}\left(I_{k} w, I_{k} w\right)=a_{k-1}(w, w), \quad \text { for all } w \in V_{k-1}
$$

for any bilinear form $a_{k}(\cdot, \cdot): V_{k} \times V_{k} \rightarrow R$. We consider the following finite element discretization equation of (1); Find $u_{J} \in V_{J}$ such that

$$
a_{J}\left(u_{J}, v_{J}\right)=\left(f, v_{J}\right), \quad \text { for all } v_{J} \in V_{J} .
$$

Let $A_{k}(k=0, \ldots, J)$ be the matrix representations of the form $a_{k}(\cdot, \cdot)$ on $V_{k} \times V_{k}$ with respect to a certain discrete inner product $(\cdot, \cdot)_{k}$. Define $P_{k-1}: V_{k} \rightarrow V_{k-1}$ by

$$
\left(A_{k-1} P_{k-1} w, v\right)_{k-1}=\left(A_{k} w, I_{k} v\right)_{k}, \quad \text { for all } v \in V_{k-1} \text { and } w \in V_{k}
$$


and the restriction operator $P_{k-1}^{0}: V_{k} \rightarrow V_{k-1}$ by

$$
\left(P_{k-1}^{0} w, v\right)_{k-1}=\left(w, I_{k} v\right)_{k}, \quad \text { for all } v \in V_{k-1} \text { and } w \in V_{k} \text {. }
$$

Now the discretization (7) can be written in the above notation as

$$
A_{J} u_{J}=f_{J}
$$

where $f_{J}$ is the vector representation of $f$. We describe the $V$-cycle multigrid algorithm of (9). Let $R_{k}$ be any smoothing operator such as the Jacobi and Gauss-Seidel iterations. Then the $V$-cycle algorithm is defined as follows:

Multigrid Algorithm. Set $B_{0}=A_{0}^{-1}$. For $k \geqslant 1$ define $B_{k}: V_{k} \rightarrow V_{k}$ in terms of $B_{k-1}$ as follows. Let $g \in V_{k}$.

1. Set $x^{0}=0$.

2. Define $x^{l}$ for $l=1, \ldots, m$ by $x^{l}=x^{l-1}+R_{k}\left(g-A_{k} x^{l-1}\right)$.

3. Set $B_{k} g=x^{m}+B_{k-1} P_{k-1}^{0}\left(g-A_{k} x^{m}\right)$.

From the definition of $P_{k-1}$, we have

$$
P_{k-1}^{0} A_{k}=A_{k-1} P_{k-1}
$$

Let $K_{k}=I-R_{k} A_{k}$. Then we have, for $m=1$,

$$
\left(I-B_{k} A_{k}\right) x=\left[\left(I-P_{k-1}\right)+\left(I-B_{k-1} A_{k-1}\right) P_{k-1}\right] K_{k} x .
$$

For the convergence analysis of the multigrid algorithm, one needs to impose some conditions on the smoother $[4,5,12]$, i.e.

1. There is a constant $C_{R}$ such that

$$
\frac{(u, u)_{k}}{\lambda_{k}} \leqslant C_{R}\left(\bar{R}_{k} u, u\right)_{k}, \quad \text { for all } u \in V_{k},
$$

where $\bar{R}_{k}=\left(I-K_{k}^{T} K_{k}\right) A_{k}^{-1}$ and $\lambda_{k}$ is the largest eigenvalue of $A_{k}$. Here the superscript "T" denotes the adjoint with respect to the inner product $a_{k}(\cdot, \cdot)$.

2. Let $T_{k}=\left(I-K_{k}\right) P_{k}$. There exists a positive constant $\theta<2$ independent of $k$ such that

$$
a_{k}\left(T_{k} u, T_{k} u\right) \leqslant \theta a_{k}\left(T_{k} u, u\right) \text { for all } u \in V_{k} .
$$

Then we have the well-known convergence theorem on the $V$-cycle multigrid algorithm $[12]$.

Theorem 3.1. Let $V_{k}$ be the piecewise linear finite element space and let the intergrid transfer operator $I_{k}$ satisfy (6), and let $R_{k}$ be any smoother satisfying (C.1) and (C.2). Then there exists a $\delta<1$ such that the following estimation holds

$$
\left|a_{J}\left(\left(I-B_{J} A_{J}\right) v,\left(I-B_{J} A_{J}\right) v\right)\right| \leqslant \delta^{2} a_{J}(v, v), \quad \text { for all } v \in V_{J} .
$$



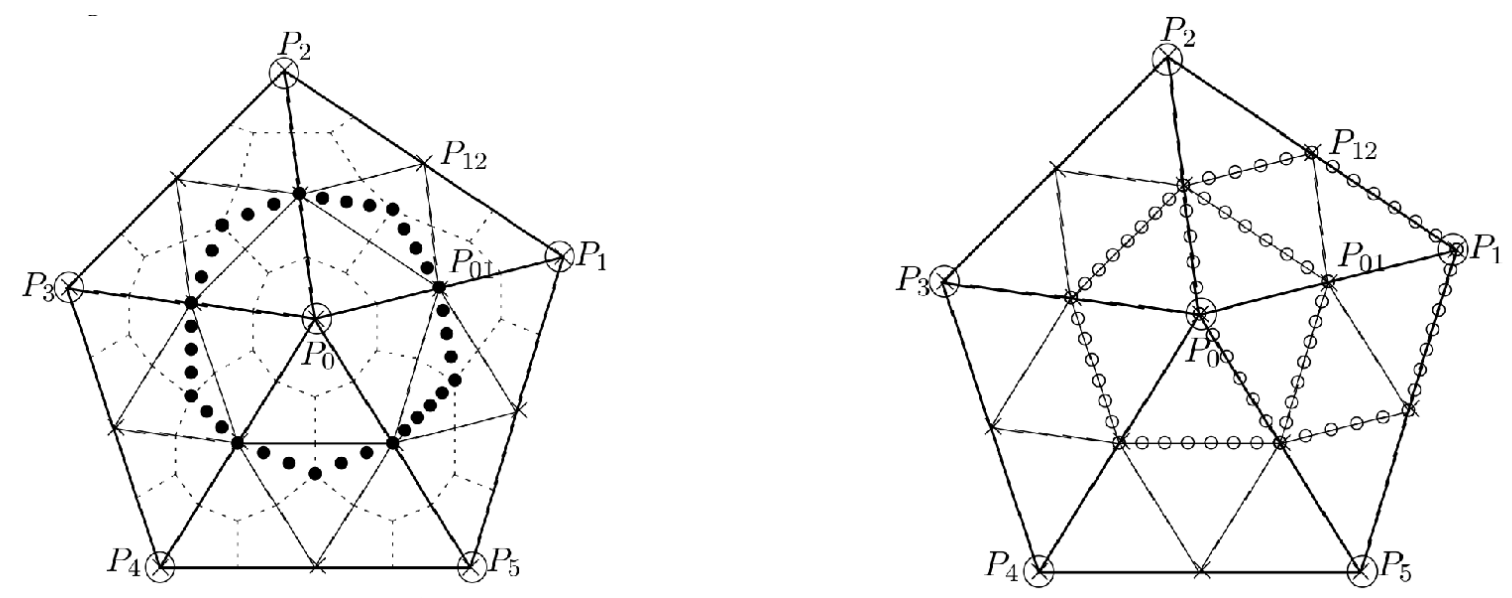

Figure 4. Control volumes of non-overlapping (left) and overlapping (right) control volume. $\times$ :fine grid vertices, o:coarse grid vertices.

Next, we consider the intergrid transfer operators based on the control volumes which might not satisfy (6). It is easily verified that the piecewise constant finite dimensional spaces $Y_{k}$ are non-nested, so we define the intergrid transfer operator $Q_{k}^{*}: Y_{k-1} \rightarrow Y_{k}$ by the following volume based equations:

$$
Q_{k}^{*} v(P)=\sum_{i=1}^{N_{k-1}} \frac{\left|K_{P_{i}}^{*} \cap K_{P}^{*}\right|}{\sum_{i=1}^{N_{k-1}}\left|K_{P_{i}}^{*} \cap K_{P}^{*}\right|} v\left(P_{i}\right),
$$

where $P$ is a node point of $\mathcal{T}_{k}$ and the $P_{i}$ are node points of $\mathcal{T}_{k-1}$.

From the following relation of function spaces $V_{k}$ and $Y_{k}$ for all $k$

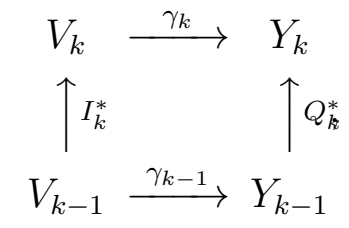

we define intergrid transfer operators $I_{k}^{*}: V_{k-1} \rightarrow V_{k}$ as $I_{k}^{*}=\gamma_{k}^{-1} Q_{k}^{*} \gamma_{k-1}$.

We consider the non-overlapping control volume based intergrid transfer operators $I_{k}^{*, n}$ : $V_{k-1} \rightarrow V_{k}$ as in figure 4 (left). From the definition of the control volume, we have

$$
\sum_{i=1}^{N_{k-1}}\left|K_{P_{i}}^{*} \cap K_{P}^{*}\right|=\left|K_{P}^{*}\right|
$$

and

$$
I_{k}^{*, n} v\left(P_{i j}\right)= \begin{cases}v\left(P_{i}\right), & \text { if } i=j \\ \alpha_{0} v\left(P_{i}\right)+\alpha_{1} v\left(P_{j}\right)+\alpha_{2} v\left(P_{i^{\prime}}\right)+\alpha_{3} v\left(P_{j^{\prime}}\right), & \text { if } i \neq j\end{cases}
$$

where $\alpha_{0}+\alpha_{1}+\alpha_{2}+\alpha_{3}=1, \alpha_{k} \geqslant 0$ for $k=0,1,2,3$, and $P_{i^{\prime}}$ and $P_{j^{\prime}}$ are the vertex points of triangles which have the vertexes $P_{i}$ and $P_{j}$.

Remark 3.1. If $Q$ is a center of triangle elements, then we easily see that $I_{k}^{*, n}$ is the same operator with the natural injection operator $I_{k}$ because $\alpha_{2}=\alpha_{3}=0$ and $\alpha_{0}=\alpha_{1}=\frac{1}{2}$. 
In the same way, we define the overlapping control volume based intergrid transfer operators $I_{k}^{*, o}: V_{k-1} \rightarrow V_{k}$ for the overlapping control volume as in figure 4 (right). Then we have

$$
I_{k}^{*, o} v\left(P_{i j}\right)= \begin{cases}\frac{1}{3} v\left(P_{i}\right)+\sum_{i^{\prime}} \alpha_{i i^{\prime}} v\left(P_{i^{\prime}}\right), & \text { if } i=j \\ \frac{1}{3}\left(v\left(P_{i}\right)+v\left(P_{j}\right)\right)+\alpha_{2} v\left(P_{i^{\prime}}\right)+\alpha_{3} v\left(P_{j^{\prime}}\right), & \text { if } i \neq j\end{cases}
$$

where the $P_{i^{\prime}}$ in the case $i=j$ are the vertex of the triangles which have the vertex $P_{i}$, $\sum_{i^{\prime}} \alpha_{i i^{\prime}}=\frac{2}{3}, \alpha_{i i^{\prime}} \geqslant 0, \alpha_{2}+\alpha_{3}=\frac{1}{3}$, and $\alpha_{2} \geqslant 0$ and $\alpha_{3} \geqslant 0$.

Remark 3.2. From the definition of $I_{k}^{*, n}$ and $I_{k}^{*, o}$, we can easily see the boundedness of the intergrid operators, i.e.,

$$
a_{k}\left(I_{k}^{*} v, I_{k}^{*} v\right) \leqslant C a_{k-1}(v, v) \text { for all } v \in V_{k-1} .
$$

with the equivalence relation of the bilinear forms. In convergence theorems of the multigrid method for the $V$-cycle in [5], the constant $C$ has to be less than two. But, the constant $C$ can be estimated only for the special cases. The convergence of the multigrid method for the $W$-cycle and the boundedness of the condition number of the variable $V$-cycle with these intergrid operators can be proved in much the same way as in [5].

Now, we focus on a convergence analysis of the $V$-cycle multigrid method. For this purpose, we adopt and use results by [12] who consider the case of the non-overlapping finite volume element method with the natural injection $I_{k}$.

We consider the bilinear form $a_{k}^{*}(\cdot, \cdot)$ of the finite volume method as a perturbed bilinear form of $a_{k}(\cdot, \cdot)$ on $V_{k} \times V_{k}$. We denote the difference between the two bilinear forms by

$$
d_{k}(w, v)=a_{k}(w, v)-a_{k}^{*}(w, v) \quad w, v \in V_{k},
$$

and assume the perturbation condition

$$
\left|d_{k}(w, v)\right| \leqslant C h_{k}\|w\|_{1}\|v\|_{1}, \quad w, v \in V_{k},
$$

for a certain $h_{0}$ which depends on the problem.

Lemma 3.1. The bilinear forms $a^{*}(\cdot, \cdot)$ based on the non-overlapping and overlapping control volumes satisfy the condition (P.1).

Proof. A proof for the non-overlapping FVM can be found in [12]. For the overlapping FVM, we have

$$
\left|d_{k}(w, v)\right| \leqslant C \sum_{K \in \mathcal{T}_{k}} h_{k}|w|_{1, K}|v|_{1, K} \leqslant C h_{k}\|w\|_{1}\|v\|_{1}
$$

by Lemma 5.2 in [9] and the equivalence of the norms.

Let $A_{k}^{*}$ be the matrix representation of $a_{k}^{*}$ with respect to $(\cdot, \cdot)_{k}$. Then, in parallel with $a_{k}(\cdot, \cdot)$, we define $R_{k}^{*}$ and $K_{k}^{*}$ using $A_{k}^{*}$ in place of $A_{k}$. These matrices $R_{k}^{*}$ and $K_{k}^{*}$ are related with a smoothing operator in the multigrid method and the assumption

$$
\left|a_{k}\left(\left(K_{k}-K_{k}^{*}\right) w, v\right)\right| \leqslant C h_{k}\|w\|_{a}\|v\|_{a}, \quad w, v, \in V_{k},
$$

used and proved in [12].

We define $P_{k-1}^{*}: V_{k} \rightarrow V_{k-1}$ by

$$
a_{k-1}^{*}\left(P_{k-1}^{*} w, v\right)=a_{k}^{*}\left(w, I_{k} v\right) \quad \text { for all } w \in V_{k}, v \in V_{k-1} .
$$

Then we have the following Lemma and theorem (see [12]) for the multigrid method for the FVE with the natural injections $I_{k}$ as the intergrid transfer operators. 
Lemma 3.2. For $w \in V_{k}, v \in V_{k-1}$, for all $k>1$, we have

$$
\left|a_{k-1}\left(P_{k-1}^{*} w, v\right)-a_{k-1}\left(P_{k-1} w, v\right)\right| \leqslant C h_{k}\|w\|_{1}\|v\|_{1} .
$$

Theorem 3.2. Assume the multigrid algorithm for the original problem (7) has the convergence property given in Theorem 3.1. Suppose that the $R_{k}^{*}$ satisfy (P.2). Then there exists an $h_{0}$ such that for all $h_{1}<h_{0}$,

$$
\left|a_{J}\left(\left(I-B_{J}^{*} A_{J}^{*}\right) v,\left(I-B_{J}^{*} A_{J}^{*}\right) v\right)\right| \leqslant\left(\delta^{*}\right)^{2} a_{J}(v, v), \quad \text { for all } v \in V_{J},
$$

where $\delta^{*}=\delta+c h_{1}<1$ and $\delta$ is as in Theorem 3.1 .

From now on, we consider the control volume based intergrid transfer operators $I_{k}^{*}$. In the same way, we define $P_{k-1}^{*}: V_{k} \rightarrow V_{k-1}$ by

$$
a_{k-1}\left(P_{k-1}^{*} w, v\right)=a_{k}\left(w, I_{k}^{*} v\right) \quad \text { for all } w \in V_{k}, v \in V_{k-1} \text {. }
$$

Remark 3.3. The operators $P_{k-1}^{*}$ in (11) and (12) are different. The multigrid operator $B_{k}^{*}$ is defined with smoother operator $R_{k}$, i.e.,

$$
I-B_{k}^{*} A_{k}=\left[\left(I-P_{k-1}^{*}\right)+\left(I-B_{k-1} A_{k-1} P_{k-1}^{*}\right)\right] K_{k} .
$$

So we don't require the assumtion (P.2).

Then we have the following lemma for $I_{k}^{*}$ based on (non-overlapping and overlapping) control volumes.

Lemma 3.3. For $w \in V_{k}, v \in V_{k-1}$, we have

$$
\left|a_{k-1}\left(P_{k-1}^{*} w, v\right)-a_{k-1}\left(P_{k-1} w, v\right)\right| \leqslant C h_{k}\|w\|_{1}\|v\|_{1} .
$$

based on (non-overlapping and overlapping) control volumes .

Proof. For $w \in V_{k}$ and $v \in V_{k-1}$, we get

$$
\begin{aligned}
& a_{k-1}\left(P_{k-1}^{*} w, v\right)-a_{k-1}\left(P_{k-1} w, v\right)=a_{k}\left(w, I_{k} v\right)-a_{k}\left(v, I_{k}^{*} v\right) \\
& \quad=a_{k}\left(w, I_{k} v\right)-a_{k}^{*}\left(w, I_{k} v\right)+a_{k}^{*}\left(w, I_{k} v\right)-a_{k}^{*}\left(w, I_{k}^{*} v\right)+a_{k}^{*}\left(w, I_{k}^{*} v\right)-a_{k}\left(w, I_{k}^{*} v\right) \\
& \quad=d_{k}\left(w, I_{k} v\right)+a_{k}^{*}\left(w, I_{k} v\right)-a_{k}^{*}\left(w, I_{k}^{*} v\right)-d_{k}\left(w, I_{k}^{*} v\right)
\end{aligned}
$$

with the definition of $P_{k-1}$ in (8) and $P_{k-1}^{*}$ in (12).

From the Lemma 3.1 and (10), we have

$$
\left|d_{k}\left(w, I_{k} v\right)-d_{k}\left(w, I_{k}^{*} v\right)\right| \leqslant C h_{k}\|w\|_{1}\|v\|_{1} .
$$

From the facts

$$
Q_{k}^{*, n}=\int_{K_{P_{i}}^{*, n}} v \mathrm{~d} x \quad \text { and } Q_{k}^{*, o}=\frac{1}{3} \int_{K_{P_{i}}^{*, o}} v \mathrm{~d} x,
$$

we can extend the domain of $Q_{k}^{*}$ to $L^{2}$ and have the following properties [9]; for any $v \in$ $H^{1}(\Omega)$,

$$
\left\|v-Q_{k}^{*} v\right\|_{0} \leqslant C h_{k}\|v\|_{1}, \quad\left\|v-\gamma_{k} v\right\|_{0} \leqslant C h_{k}\|v\|_{1} .
$$


Let $q=\gamma_{k-1} v$, then we have,

$$
\begin{aligned}
\mid a_{k}^{*}\left(w, I_{k} v\right) & -a_{k}^{*}\left(w, I_{k}^{*} v\right)|=| b_{k}^{*}(w, q)-b_{k}^{*}\left(w, Q_{k}^{*} q\right) \mid \\
& =\left|\sum_{K_{P}^{*} \in \mathcal{T}_{k}^{*}} \int_{\partial K_{P}^{*}} \mathbb{A} \nabla w \cdot \mathbf{n} q(P) d \sigma-\sum_{K_{P}^{*} \in \mathcal{T}_{k}^{*}} \int_{\partial K_{P}^{*}} \mathbb{A} \nabla w \cdot \mathbf{n}\left(Q_{k}^{*} q\right)(P) d \sigma\right| \\
& =\left|\sum_{K_{P}^{*} \in \mathcal{T}_{k}^{*}} \sum_{K_{P_{i}}^{*} \in \mathcal{T}_{k-1}} \int_{\partial\left(K_{P}^{*} \cap K_{P_{i}}^{*}\right)} \mathbb{A} \nabla w \cdot \mathbf{n}\left(q-Q_{k}^{*} q\right) d s\right| \\
& =\left|\sum_{K_{P}^{*} \in \mathcal{T}_{k}^{*}} \sum_{K_{P_{i}}^{*} \in \mathcal{T}_{k-1}} \int_{K_{P}^{*} \cap K_{P_{i}}^{*}} \nabla \cdot \mathbb{A} \nabla w\left(q-Q_{k}^{*} q\right) d x\right| \\
& \leqslant C|\mathbb{A}|_{1, \infty}\|w\|_{1}\left\|q-Q_{k}^{*} q\right\|_{0} \leqslant C\|w\|_{1}\left\|\gamma_{k-1} v-Q_{k}^{*} \gamma_{k-1} v\right\|_{0}
\end{aligned}
$$

From (16) and the boundedness of $Q_{k}^{*}$ on any Sobolev norms, we deduce

$$
\begin{aligned}
\left\|\gamma_{k-1} v-Q_{k}^{*} \gamma_{k-1} v\right\|_{0} & \leqslant\left\|v-\gamma_{k-1} v\right\|_{0}+\left\|v-Q_{k}^{*} v\right\|_{0}+\left\|Q_{k}^{*} v-Q_{k}^{*} \gamma_{k-1} v\right\|_{0} \\
& \leqslant C h_{k}\|v\|_{1}+\left\|v-\gamma_{k-1} v\right\|_{0} \leqslant C h_{k}\|v\|_{1}
\end{aligned}
$$

i.e.,

$$
\left|a_{k}^{*}\left(w, I_{k} v\right)-a_{k}^{*}\left(w, I_{k}^{*} v\right)\right| \leqslant C h_{k}\|w\|_{1}\|v\|_{1} .
$$

From (14), (15), and (17), we have (13).

By using the Lemma 3.3, we can prove Theorem 3.2 for the multigrid method of the bilinear form $a_{k}(\cdot, \cdot)$ with the intergrid transfer operator $I_{k}^{*}$. Futhermore, we know that Theorem 3.2 is satisfied for the multigrid method of the bilinear form $a_{k}^{*}(\cdot, \cdot)$ with the intergrid transfer operator $I_{k}^{*}$.

\section{Numerical results and scaling properties}

In this section, we report numerical experiments on the convergence behavior of the finite volume multigrid methods and the scaling properties on a high performance computer.

The HPC-FF machine was dedicated to the European Fusion community and located at Jülich Supercomputing Centre (JSC), Germany. It was made by 1080 computer nodes of two Intel Xeon X5570 (Nehalem-EP) quad-core processors with 24 GB memory (DDR3, $1066 \mathrm{MHz}$ ) and connected by Infiniband Mellanox ConnectX QDR HCA. So it had 8640 cores in total and 87.3 Tera Flops Linpack performance.

For numerical experiments, we consider three different triangles: a right triangle whose circumcenter lies on the longest edge, a regular triangle whose circumstance and centroid are coincident, and a general triangle. So we consider the three following triangulations; a uniform right triangulation of the unit square domain as depicted in figure 5 (left), an uniform regular triangulation of the regular hexagon domain as depicted in figure 5 (middle), and an unstructured triangulation of the square domain as depicted in figure 5 (right). As test problems, we choose $\mathbb{A}$ to be the identity matrix.

In the numerical experiment, we use two pre-and post-smoothings in the multigrid algorithm. As a smoothing operator, we use the Gauss-Seidel iteration which is well-known and simple to implement. In Section 3, we estimated an error reduction factor $\delta^{*}$ with respect 

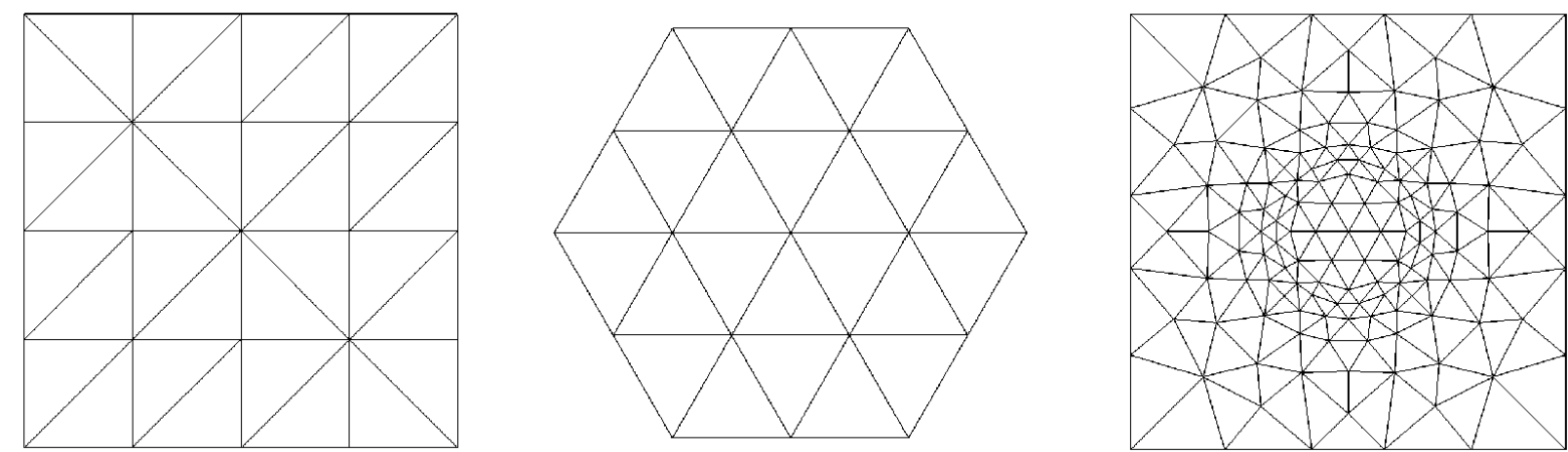

Figure 5. Domains and their triangulations: unit square(left), hexagon (middle), unstructured (right).

to the energy norm. But, we need to know the exact solution to get such error reduction factor. Instead, we use a residual error $r=b-A x$ which can be computed easily without the exact solution in implementation. We consider an average error reduction factor $\bar{\delta}_{n}$,

$$
\bar{\delta}_{n}=\left(\frac{\left\|r_{n}\right\|_{0}}{\left\|r_{0}\right\|_{0}}\right)^{\frac{1}{n}}, \quad r_{k}=B-A x_{k}, \quad \text { for } k=0, \ldots, n
$$

Also, we know that a required number of iterations $n$ is related with the average error reduction factor to reach a given relative residual error, i.e., $\left\|r_{n}\right\|_{0} \leqslant 10^{-10}\left\|r_{0}\right\|_{0}$,

$$
n=-10 \log \bar{\delta}_{n}
$$

On each discretized domain, we consider three different solvers, the V-cycle multigrid method as a solver (MG), the preconditioned conjugated gradient method (PCGM) with the V-cycle multigrid preconditioner (CGM), and the preconditioned restart GMRES with the V-cycle multigrid preconditioner (GMRES). The CGM method can only be used for symmetric and positive definite systems and the PCGM has to use the energy norm and the multigrid preconditioner has to be symmetrical. The GMRES method can be used for any system which includes non-symmetric or non-positive definite systems, but it requires more memory, so we use the restart GMRES method which does not guarantee convergence, but converges for most of the problems.

Among many non-overlapping control volumes, we consider the circumcenter based control volume because the intergrid transfer operators with the centroid coincide with the natural injections. We tested three intergrid transfer operators, namely, the natural injections $I_{k}$, the non-overlapping intergrid transfer operators $I_{k}^{*, n}$ with the circumcenter, and the overlapping intergrid transfer operators $I_{k}^{o}$. For the discretization with regular triangulation (for the regular hexagonal domain), two intergrid transfer operators $I_{k}$ and $I_{k}^{*, n}$ are the same. So the results for only two intergrid transfer operators are reported for the hexagonal domain. For the unit square domain, we have

$$
I_{k}^{*, n} v\left(P_{i j}\right)=\left\{\begin{array}{ll}
v\left(P_{i}\right), & \text { if } i=j \\
\frac{1}{2} v\left(P_{i}\right)+\frac{1}{2} v\left(P_{j}\right), & \text { if } i \neq j \text { and on edge with parallel to } x \text { - or } y \text {-axis } \\
\frac{1}{4} v\left(P_{i}\right)+\frac{1}{4} v\left(P_{j}\right)+\frac{1}{4} v\left(P_{i^{\prime}}\right)+\frac{1}{4} v\left(P_{j^{\prime}}\right), \quad \text { if } i \neq j \text { and on slanted edge }
\end{array},\right.
$$


i.e., the same with the bilinear finite element multigrid on a square mesh. For the case with same areas for all discretizations, such as the unit square domain and the regular hexagonal domain, we have

$I_{k}^{*, o} v\left(P_{i j}\right)=\left\{\begin{array}{l}\frac{1}{3} v\left(P_{i}\right)+\sum_{i^{\prime}} \frac{2}{3 m} v\left(P_{i^{\prime}}\right), \text { if } i=j \text { and } m \text { is the number of neighboring vertexes } \\ \frac{1}{3} v\left(P_{i}\right)+\frac{1}{3} v\left(P_{j}\right)+\frac{1}{6} v\left(P_{i^{\prime}}\right)+\frac{1}{6} v\left(P_{j^{\prime}}\right), \quad \text { if } i \neq j\end{array}\right.$

We depict the average error reduction factor with the required number of iterations in figure 6 and the solution times in figure 7 . We use different symbols for three intergrid transfer operators, i.e., the solid line for $I_{k}, \diamond \diamond \diamond$ for $I_{k}^{*, n}$, and $\bullet \bullet \bullet$ for $I_{k}^{*, o}$.

First, we depict the average error reduction factor according to the number of levels when the finest level is fixed. The numerical results show that the required number of iterations is bounded and does not increase rapidly. The non-overlapping covolume based intergrid transfer operator $I_{k}^{*, n}$ has different behaviors depending on the triangulation, i.e., it gave the best performance for the unit square domain, the same for the hexagon domain, and the worst performance for the unstructured triangulation. $I_{k}$ is better than the overlapping covolume based intergrid transfer operator $I_{k}^{*, o}$.

Next, we investigated the weak scaling property of the multigrid method. It is hard to fix the number of operations per core for the multigrid method because the number of operations increases in different rates when the number of the degrees of freedom (DoF) of the finest level is increased. So we considered its semi-weak scaling property, i.e., the number of the DoF per core on the finest level is fixed. We tested a problem with 131,000 DoF per core and depicted it in figure 7.

The solution times of the solvers with $I_{k}^{*, n}$ are the fastest on the unit square domain and slower than the solvers with $I_{k}$ on the unstructured triangulation. The multigrid method with $I_{k}^{*, o}$ is the slowest solver and has poorer weak scaling properties than with $I_{k}$ and $I_{k}^{*, n}$ for all three cases. The numerical results in figure 7 show that the multigrid methods with $I_{k}$ and $I_{k}^{*, n}$ have a good weak scaling property up to 1000 cores for all cases.

\section{Conclusions}

We considered two different intergrid transfer operators based on non-overlapping and overlapping control volumes on three different triangular meshes and compared with the one based on the trial function space. The multigrid method with the intergrid transfer operator based on the trial function space has the best convergence rate, except on the uniform mesh on which the multigrid method with the non-overlapping control volume based intergrid transfer operator has a better convergence rate. We also showed that the multigrid method with these intergrid transfer operators has a good weak scaling property up to 1000 cores for all cases.

\section{Acknowledgements:}

I would like to thank R. Hatzky and the other HLST team members, as well as B. Scott (IPP) for helpful discussions.

\section{Funding:}

This project has received funding from the European Unions Horizon 2020 research and innovation programme under grant agreement number 633053. The views and opinions 
Error reduction

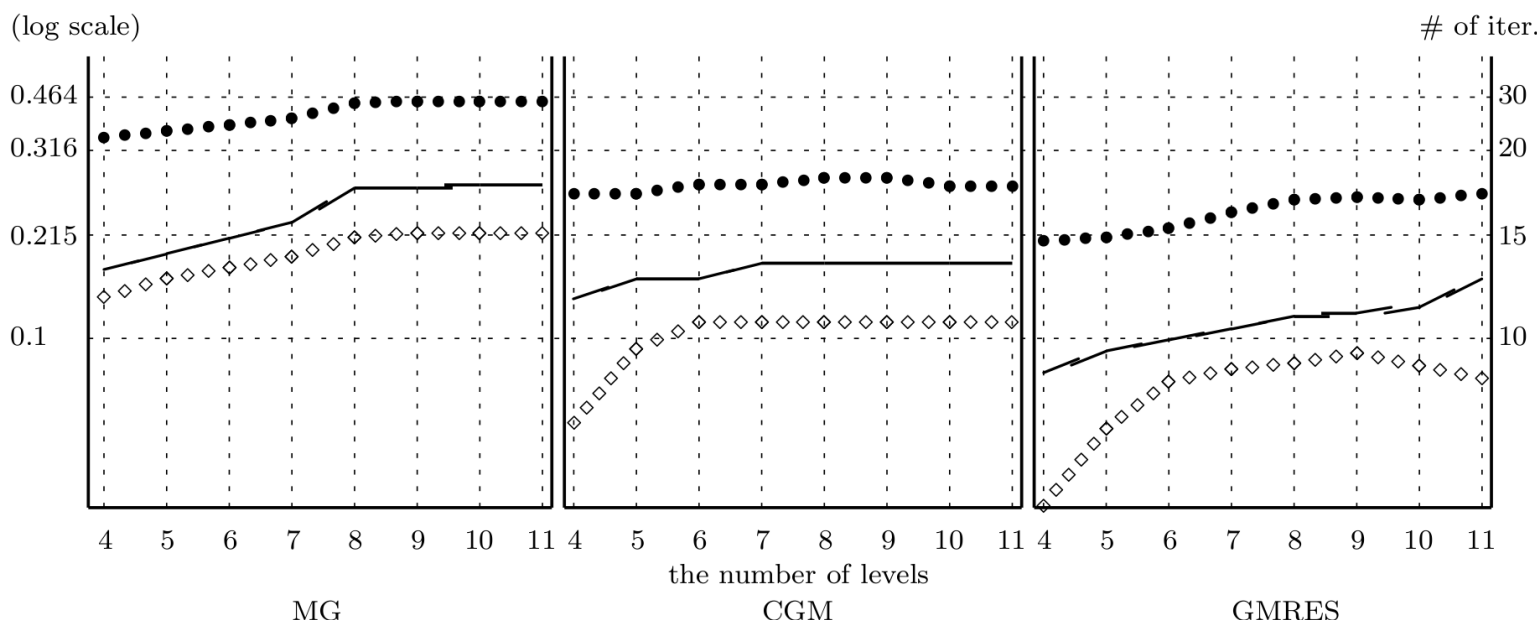

(a) Unit square

Error reduction



Error reduction

(b) Hexagon
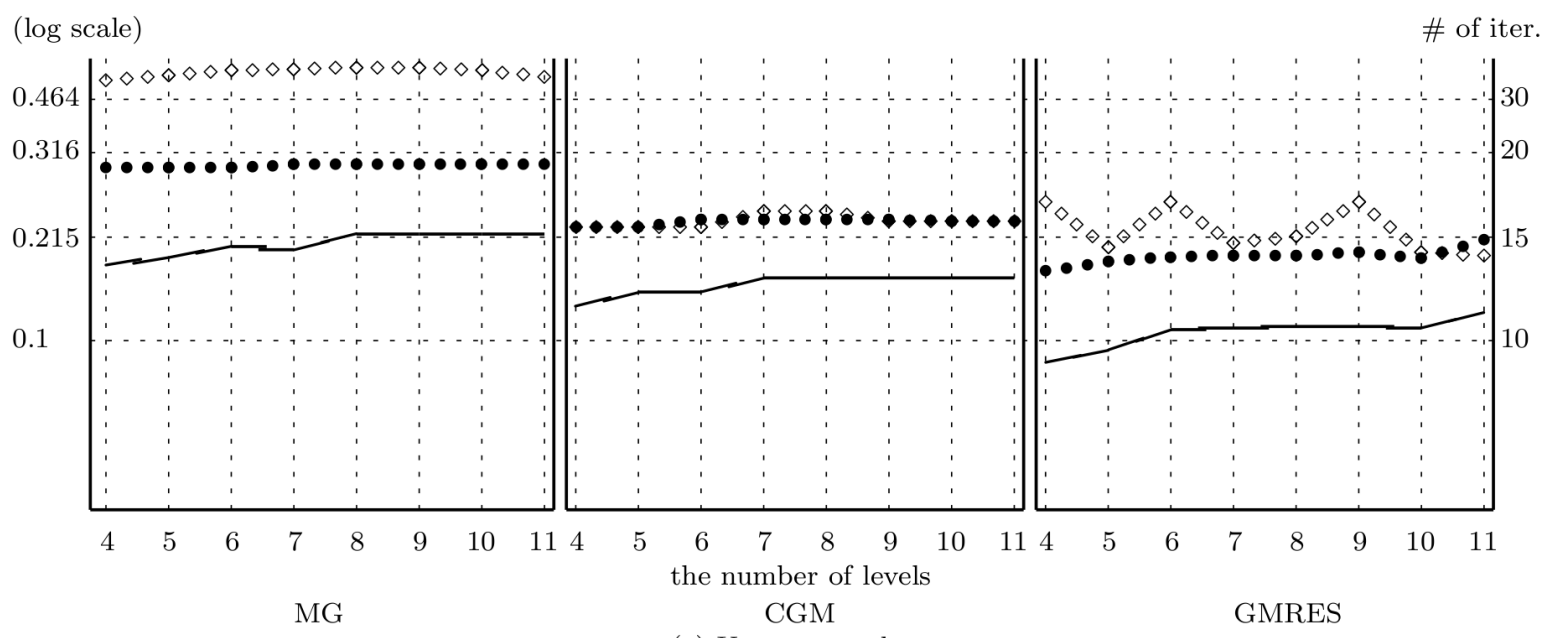

(c) Unstructured

Figure 6. The average error reduction of different solvers and the required number of iterations to get a given residual error according to the number of levels for a fixed finest grid. 




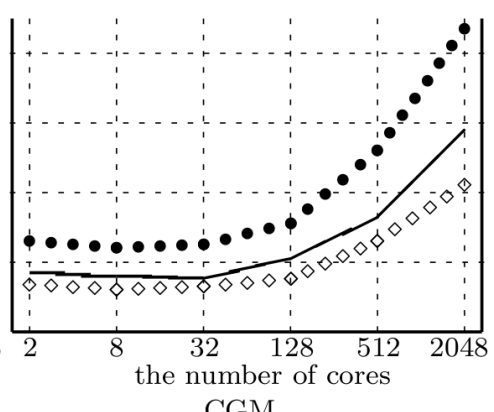

CGM

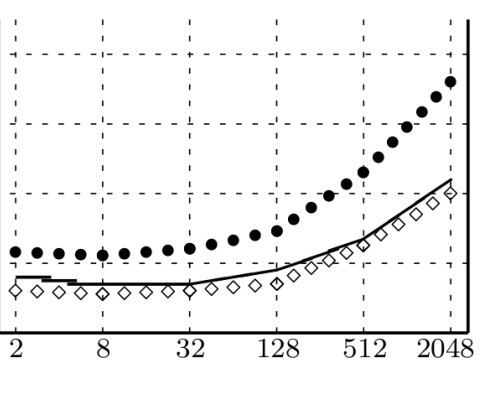

GMRES

(a) Unit square

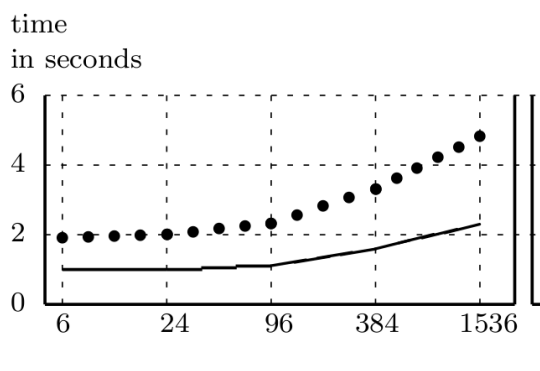

MG

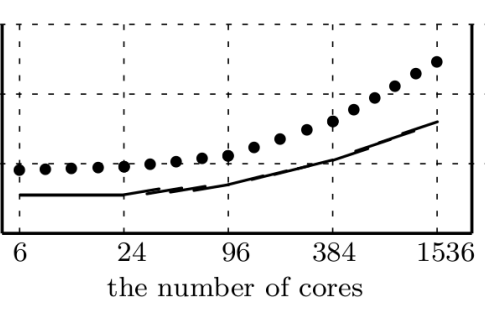

CGM

(b) Hexagon

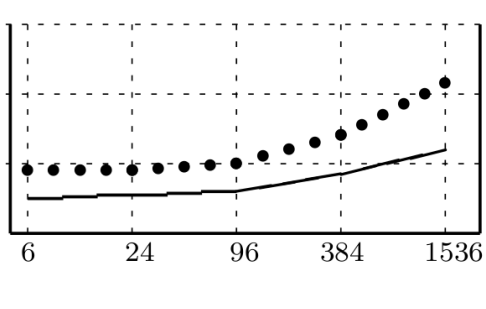

GMRES

time

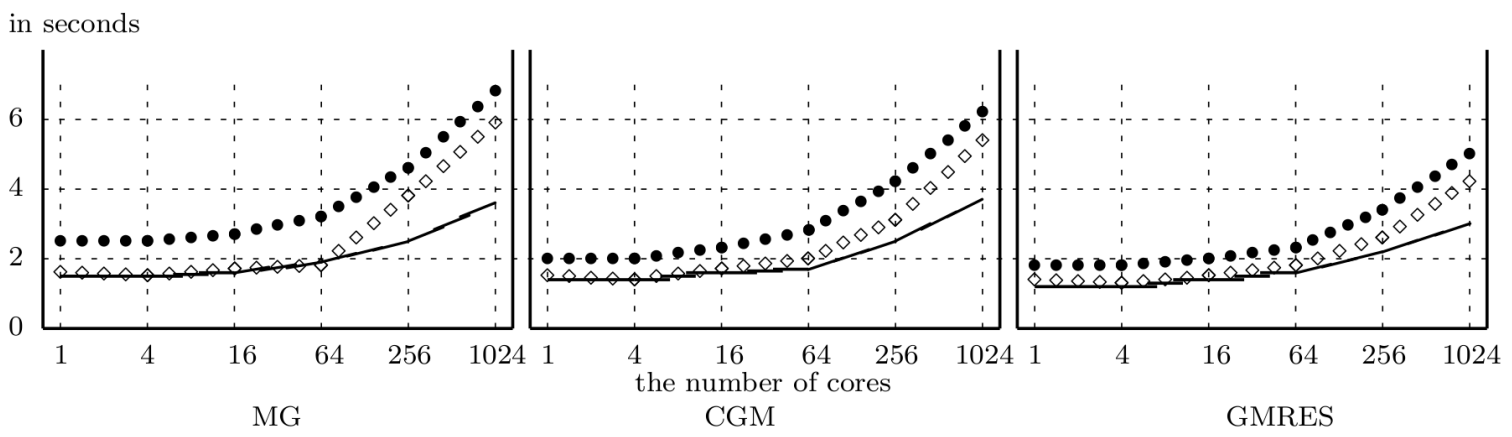

(c) Unstructured

Figure 7. The solution times as a function of the number of cores to solve the test problem with a fixed number of 131,000 DoF per core. 
expressed herein do not necessarily reflect those of the European Commission.

\section{References}

[1] N. S. Bachvalov, On the convergence of a relaxation method with natural constraints on the elliptic operator, USSR Comp. Math. and Math. Phys., 6 (1966), pp. 101-135.

[2] R. Bank and T. Dupont, An optimal order process for solving finite element equations, Math. Comp., 36 (1981), pp. 35-51.

[3] D. Braess and R. Verfürth, Multigrid methods for nonconforming finite elements methods, SIAM J. Numer. Anal., 27 (1990), pp. 979-986.

[4] J. Bramble, Multigrid Methods, Pitman, London, 1993.

[5] J. Bramble, J. Pasciak, and J. Xu, The analysis of multigrid algorithms with non-nested spaces or noninherited quadratic forms, Math. Comp., 56 (1991), pp. 1-34.

[6] A. Brandt and O. E. Livne, Multigrid techniques: 1984 Guide with Applications to Fluid Dynamics, Revised Edition, Classics Appl. Math. 67, SIAM, Philadelphia, 2011.

[7] Z. Cai, On the finite volume element method, Numerische Mathematik, 58 (1991), pp. 713-735.

[8] Z. Cai, J. Mandel, and S. McCormick, The finite volume element method for diffusion equations on general triangulations, SIAM J. Numer. Anal., 28 (1991), pp. 392-402.

[9] P. Chatzipantelidis, Finite Volume Methods for Elliptic PDE's: A New Approach, Mathematical Modelling and Numerical Analysis, 36 (2002), pp. 307-324.

[10] S. H. Chou, Analysis and convergence of a covolume method for the generalized Stokes Problem, Math. Comp. 66 (1989), pp. 85-104.

[11] S. H. Chou and Q. Li, Error estimates in $l^{2}, H^{1}$ and $L^{\infty}$ in covolume methods for elliptic and parabolic problems: a unified approach, Math. Comp. 209 (2000), pp. 103-120.

[12] S. H. Chou and D. Y. Kwak, Multigrid algorithms for a vertex-centered covolume method for elliptic problems, Numer. Math. 90 (2002), pp. 441-458.

[13] S. H. Chou and X. Ye, Unified analysis of finite volume methods for second order elliptic problems, SIAM J. Numer. Anal., 45 (2007), pp. 1639-1653.

[14] R. P. Fedorenko, A relaxation method for solving elliptic difference equations, USSR Comp. Math. and Math. Phys., 1 (1962), pp. 1092-1096.

[15] W. Hackbusch, Multigrid Methods and Applications, Springer-Verlag, Berlin, Germany, 1985.

[16] K. S. Kang, Covolume-based intergrid transfer operator in $P_{1}$ nonconforming multigrid method, Applied Numerical Mathematics, 51 (2004), pp. 47-67.

[17] K. S. Kang, $P_{1}$ nonconforming finite element method for radiation transport, SIAM J. Sci. Comput., 25 (2003), pp. 369-384.

[18] K. S. Kang and D. Y. Kwak, Error Estimate in $L^{2}$ of a covolume method for the generalized Stokes problem, Numer. Methods Partial Differential Eqs., 22 (2006), pp. 165-179.

[19] R. Sadourny, A. Arakawa, and Y. Mintz, Integration of the nondivergent barotropic vorticity equation with an Icosahedral-Hexagonal grid for the sphere, Monthly Weather Review, 96 (1968), pp. 351-356.

[20] P. Wesseling, An Introduction to Multigrid Methods, Wiley, Chichester, 2003.

[21] P. Wesseling and M. Khalil, Vertex-centered and cell-centered multigrid for interface problems, Journal of Computational Physics, 98 (1992), pp. 1-10. 